\title{
The Prevalence of Chronic Obstructive Pulmonary Disease (COPD) and the Heterogeneity of Risk Factors in the Canadian Population: Results from the Canadian Obstructive Lung Disease (COLD) Study
}

This article was published in the following Dove Press journal:

International Journal of Chronic Obstructive Pulmonary Disease

\begin{abstract}
Clarus Leung, (D' Jean Bourbeau, ${ }^{2}$ Don D Sin, (D)'Shawn D Aaron, ${ }^{3}$ J Mark FitzGerald, ${ }^{4}$

François Maltais, (iD ${ }^{5}$

Darcy D Marciniuk, ${ }^{6}$

Denis O'Donnell, ${ }^{7}$ Paul Hernandez, ${ }^{8}$

Kenneth R Chapman, (D) ${ }^{9}$

Brandie Walker, ${ }^{10}$ Jeremy D Road, ${ }^{4}$

Liyun Zheng,' Carl Zou,

James C Hogg,' Wan C Tan (D)'

On behalf of the CanCOLD

Collaborative Research Group

'Centre for Heart Lung Innovation, St Pauls Hospital, The University of British Columbia, Vancouver, BC, Canada; ${ }^{2}$ Research Institute McGill University Health Centre, McGill University, Montreal, Quebec, Canada; ${ }^{3}$ The Ottawa Hospital Research Institute, University of Ottawa, Ottawa, Canada; ${ }^{4}$ Department of Medicine, Vancouver General Hospital,

University of British Columbia, Vancouver,

Canada; Institut Universitaire de Cardiologie

et de Pneumologie de Québec, Université Laval,

Quebec City, Quebec, Canada; ${ }^{6}$ Respiratory

Research Centre, University of Saskatchewan,

Saskatoon, Canada; ' ${ }^{7}$ Department of Medicine,

Queen's University, Kingston, Canada;

${ }^{8}$ Department of Medicine, Dalhousie

University, Halifax, Canada; ${ }^{9}$ Toronto

General Hospital Research Institute,

University of Toronto, Toronto, Canada;

${ }^{10}$ Department of Medicine, University of

Calgary (BW), Alberta, Canada
\end{abstract}

Correspondence: Wan C Tan

Centre for Heart Lung Innovation,

St. Paul's Hospital, University of British

Columbia, Rm I66, I08I Burrard Street,

Vancouver, B.C, V6Z IY6, Canada

Tel + I-604-682-2344 ext 62749

$\mathrm{Fax}+\mathrm{I}-604-806-9274$

Email wan.tan@hli.ubc.ca
Purpose: To determine the spirometric-based prevalence of COPD across different regions in Canada and to evaluate the site heterogeneity of risk factors.

Patients and Methods: In this cross-sectional, population-based study, random samples of non-institutionalized adults aged $\geq 40$ years were generated by random digit dialling. Participants answered an interviewer-administered questionnaire and performed spirometry before and after bronchodilator administration. COPD was defined as post-bronchodilator $\mathrm{FEV}_{1} / \mathrm{FVC}<0.70$ (fixed ratio, $\mathrm{FR}$ ) and $\mathrm{FEV}_{1} / \mathrm{FVC}<5$ th percentile (lower limits of normal, LLN). Separate logistic regression models were used to compute the risk (adjusted odds ratio, aOR) for COPD. $\mathrm{I}^{2}$ and $\mathrm{Tau}^{2}$ analyses were used to evaluate heterogeneity.

Results: Out of 5176 (95\%) participants, 4893 (47\% male with mean age 56.6 years (95\% confidence interval, 56.0-57.2)) had spirometry that satisfied ATS criteria. The population prevalence of COPD was 16.2\% (95\% CI, 14.5-17.8) by FR and 11.2\% (95\% CI, 9.7-12.6) by LLN. Male predominance in prevalence was shown by FR but not by LLN criteria. Patient characteristics associated with an increased risk of COPD included: age (OR 1.56; 95\% CI 1.33-1.84); history of physician-diagnosed asthma (OR 3.30; 95\% CI 2.42-4.49); and childhood hospitalization for respiratory illness (OR 1.81; 95\% CI 1.17-2.80). In terms of smoking-related risk factors, current smoking status had the highest odds ratio (OR 3.49; 95\% CI 2.55-4.80). Variance in prevalence among sites was significantly reduced by adjusting for risk factors in $\mathrm{Tau}^{2}$ analyses. Higher odds of exposure for each risk factor was found in more severe COPD, suggesting that a higher risk could be linked to the development of severe disease.

Conclusion: This study reports the population prevalence of COPD in nine urban cities which collectively represent the majority of the Canadian population and demonstrates that heterogeneity in prevalence among sites is substantially explained by variation in associated risk factors for COPD.

Keywords: prevalence, heterogeneity, COPD

\section{Introduction}

The burden of COPD in Canada and worldwide is substantial and increasing. ${ }^{1}$ The Global Burden of Disease study estimated that the projection of global increase in COPD from 1990 to 2020 will rise from sixth to third in terms of morbidity. ${ }^{2}$ Chronic respiratory diseases collectively account for $4.7 \%$ of global disabilityadjusted life year, with COPD comprising two-third of this total. ${ }^{3}$ In addition, 
COPD has a significant impact on healthcare resource utilization and workplace productivity loss. Previous results of Canadian economic analysis from 2003 demonstrated that the annual direct cost of COPD-related primary and secondary care visits, treatments, and laboratory tests was at almost \$2000 CAD per patient, with unscheduled care visits accounting for almost $60 \%$ of the total direct cost of COPD. ${ }^{4}$

Spirometry is the most widely available, reproducible test of lung function and is essential to the diagnosis of COPD and reduces underdiagnosis. ${ }^{5}$ Population-based data suggested that the prevalence of COPD confirmed by spirometry is 2.5 times higher than the rate for selfreported diagnosis and fourfold higher than the previous estimates based on annual community surveys. ${ }^{6,7}$

Insight regarding the impact of variations in risk factors on COPD is important in interpreting prevalence of this disease. The relationship between tobacco smoking and the development of COPD is well established. The rate of daily or occasional smoking in Canada has decreased from $25.9 \%$ to $19.9 \%$ between 2001 and $2011,{ }^{8}$ yet the prevalence of COPD continues to increase. A decline in smoking may not have an immediate and parallel impact on the prevalence in COPD. As COPD is the result of long-term cumulative exposure to noxious gas and particles such as in tobacco smoking, a time lag between a fall in the prevalence of risk factors and prevalence in COPD is to be expected. Other reasons for a rising COPD prevalence include improved diagnosis and an aging population as the incidence of COPD peaks in older adults.

There is little information on the heterogeneity of risk factors within a country and its impact on the variation in COPD prevalence across different regions. ${ }^{6}$ Understanding the impact of variations in risk factors on COPD is important in interpreting the prevalence of this disease. Our working hypothesis was that much of the apparent heterogeneity among large urban centers in the same country, with similar health-care delivery, might be explained by differences in demographics (eg, age) and other risk factors such as asthma. It is also unclear whether the association between risk factors and COPD varies with disease severity.

To address these issues, the Canadian Obstructive Lung Disease (COLD) Study, a pan-Canadian initiative was formed to measure COPD across the nation with similar sampling strategy and standardized methods as that of the International Burden of Obstructive Lung Disease
(BOLD) $)^{9,10}$ and the PLATINO initiatives ${ }^{11}$ with the aim to provide accurate population-based estimates of COPD prevalence and risk factors. The study recruited adults aged $\geq 40$ years through random digit dialling from nine Canadian urban city sites and collected demographic and clinical information, including spirometry before and after bronchodilation, between 2006 and 2011. ${ }^{6}$

The objective of this cross-sectional multisite, nationwide, population-based study was to determine the estimated population prevalence of COPD as defined by two widely used spirometric definitions (FR and LLN), in nine Canadian cities; to evaluate the heterogeneity of risk factors associated with the disease across sites; and to assess whether the variation in prevalence for COPD between site can be reduced by adjustment for common risk factors.

\section{Methods}

\section{Study Population and Design}

Data for this cross-sectional, multisite, nationwide, population-based study were collected between 2006 and 2011 in 9 sites across Canada. The overall sampling strategy and baseline study protocol of the COLD study were the same as that used in the BOLD initiative, the full details of which have been published previously. ${ }^{6,9,10}$ Random samples of non-institutionalized adults aged $\geq 40$ years in nine Canadian urban sites in six provinces (Vancouver, Montreal, Toronto, Halifax, Calgary, Ottawa, Kingston, Quebec City, Saskatoon) were drawn as in the BOLD protocol, ${ }^{9}$ with each sampling frame consisting of a population of at least 150,000 individuals of all ages. Using landline telephone digit dialling to identify eligible individuals, random samples were created by BCStats (Victoria, BC, Canada) using official data from Statistics Canada (Survey and Analysis Section, Victoria, BC, Canada) and recruitment was conducted by NRG Research Group (Vancouver, Canada) at each respective site. Eligible individuals were invited to attend a visit to complete an interviewer-administered respiratory questionnaire and to perform pre- and post-bronchodilator spirometry by trained research staff.

\section{Study Questionnaire and Spirometric Measurements}

Trained and certified technicians administered questionnaires in English or French to participants. The standardized questionnaire included demographic details, information on respiratory symptoms and diseases, 
smoking history (tobacco and marijuana) and other risk factors for COPD, medication use, health-care utilization, activity limitation, health-related quality of life, and occupational and biomass exposure. Lung function data were obtained in the seated position with the EasyOne spirometer (ndd Medical Technologies Inc., Andover, MA, USA) before and 15 minutes after administration of two puffs $(200 \mu \mathrm{g})$ of salbutamol via a metered-dose inhaler with a spacer. Pulmonary function quality assurance with over reading was conducted, in which all spirograms were reviewed and graded using American Thoracic Society (ATS) and European Respiratory Society (ERS) guidelines with prompt feedback to the technician at each site. ${ }^{12}$ All sites used the same scoring criteria and protocol. The quality of all test sessions was reviewed centrally in the COLD study pulmonary function reference centre in Vancouver. Only spirometric data that fulfilled the ATS/ ERS acceptability and repeatability criteria were used for analysis (at least two acceptable and reproducible tests for both forced expiratory volume in 1 second $\left[\mathrm{FEV}_{1}\right]$ and forced vital capacity $[\mathrm{FVC}]) .^{13}$

\section{Definitions of Airflow Limitation}

Chronic airflow limitation was defined, a priori, as postbronchodilator $\mathrm{FEV}_{1} / \mathrm{FVC}<0.70$ or fixed ratio (FR). The severity of airflow limitation was categorized according to the Global Initiative for Chronic Obstructive Lung Disease (GOLD) stages, grades $1-4 .{ }^{14}$ Our study also computed the prevalence of COPD as defined by the lower limits of normal (LLN) of $\mathrm{FEV}_{1} / \mathrm{FVC}$, an alternative spirometric definition for airflow limitation. ${ }^{9,11,14-16}$

\section{Consent}

All participants gave written informed consent, and the study was approved by all respective University and Institutional Ethical Review Boards: UBC/PHC Research Ethics Board, P05-006 (Vancouver); Biomedical-C Research Ethics Board, BMC-06-002 (Montreal); UHN REB, 06-0421-B (Toronto); Capital Health Research Ethics Board, CDHA-RS/2007-255 (Halifax); Conjoint Health Research Ethics Board, ID21258 (Calgary); DMED-1240-09 (Kingston); 2009519-01H (Ottawa); BioREB09-162 (Saskatoon); CER20459 (Quebec City).

\section{Statistical Analysis}

All data analyses were performed using statistical software (SAS version 9.4, SAS Institute, Cary, NC, USA), and all tests were two-tailed in nature; $p \leq 0.05$ was considered significant. Univariate analysis of variables across sites was performed using one-way analysis of variance for continuous variables or the $X^{2}$-test for dichotomous variables.

The prevalence data in this study were weighted to the sex and age profile of the Canadian population. Estimated population prevalence was shown as \% (95\% CI). Sample prevalence was converted to site-specific population prevalence by adjusting for age and sex (weighted) to that of the local site population. The details for each site population were obtained from the Canadian population (Census 2006). The population estimates were shown by site and sex, and COPD was defined spirometrically in two ways: 1) by the Global Initiative Obstructive Lung Disease GOLD definition, ${ }^{14}$ that is $\mathrm{FEV} 1 / \mathrm{FVC}<0.7$ (as well as different cut-offs of lung function: $\mathrm{FEV}_{1} / \mathrm{FVC}<0.7$ and $\mathrm{FEV}_{1 \%}$ predicted $\geq 80 \%$ (GOLD grade 1 ); $\mathrm{FEV}_{1} / \mathrm{FVC}<0.7$ and $\mathrm{FEV}_{1 \%}$ predicted $<80 \%$ (GOLD grades 2 to 4 ); and 2) by less than the lower limit of normal (LLN) of $\mathrm{FEV}_{1}$ $/ \mathrm{FVC}\left(\mathrm{FEV}_{1} / \mathrm{FVC}<5\right.$ th percentile $\left[\right.$ predicted $\mathrm{FEV}_{1} / \mathrm{FVC}$ minus $1.645 \times$ standard error of estimate, SEE]). ${ }^{17,18}$

Separate logistic regression models were constructed to evaluate risk factors independently for COPD which included age, sex, years of education, smoking status, history of physician-diagnosed asthma, and childhood hospitalizations for respiratory illnesses and exposure to dusty jobs. A separate random effects meta-analysis model was constructed for each risk factor to estimate odds ratios and to assess heterogeneity by sites and by sex with use of the $\mathrm{I}^{2}$ measures. ${ }^{19}$ Individual sites were weighted according to the precision of the estimate at that site and these weights were reflected in the forest plots. The odds ratios shown for each risk factor were adjusted for age and school years, with additional adjustment as follows: 1) for asthma: packyears, childhood hospitalization, and dusty job exposure; 2) for childhood hospitalization: pack-years and dusty jobs; 3) for pack-years: asthma, childhood hospitalization, and dusty job exposure; 4) for ever smoking: asthma, childhood hospitalization, and dusty job exposure; and 5) for current smoking status: asthma, childhood hospitalization, and dusty job exposure. Further details of weightage are provided in the supplemental material. The forest plot analyses used the metan procedure in Stata V.10.0 (Stata Corp).

To show whether the variation in prevalence for COPD between site is substantially reduced by adjustment for risk factors in this study, we conducted an additional randomeffects meta-analysis analysis ${ }^{19}$ to assess the change in the variation of the prevalence for LLN COPD among the nine 
sites (heterogeneity) by adjustment for factors such as age, smoking, asthma and childhood hospitalization using two logistic regressions with random intercept (Glimmix procedure in SAS). The first model did not include any risk factors; the second multiple model included the set of risk factors; and the mean difference for the tau-squared $\left(\mathrm{Tau}^{2}\right)$ and 95\% CI was estimated using 100 bootstrap samples. Additional details of this method are in the supplemental material.

\section{Results}

\section{Participant Demographics and Risk Factors}

Table 1 shows the characteristics of the participants from the nine sites recruited between 2006 and 2011. A total of 5176 participants completed the study, comprising $47 \%$ men with mean age of 56.6 years (Table 1), of which 4893 (95\%) individuals had spirometry results that satisfied ATS/ERS criteria. The mean study response rate was $78 \%$ (ranged $63 \%$ to $88 \%$ across the 9 sites, e-Figure 1 ). The proportion of individuals aged 40 years and older in the Canadian population increased slightly from $49.34 \%$ to $51.60 \%$ between the 2006 and 2016 Canadian Census (e-Figure 2A). The proportions of non-Hispanic white and non-white ethnic groups in this study were $88.5 \%$ and $11.5 \%$, respectively (e-Figure $2 \mathrm{~B}$ ).

As shown in Table 1, the frequency of current smoking was lowest in Ottawa (11.1\%) and highest in Montreal $(20.0 \%$, ) with the heaviest tobacco exposure indicated by the number of pack-years (25.9) for Montreal. Childhood hospitalization for respiratory illness was most common in Vancouver and Kingston, $8.2 \%$ and $7.3 \%$, respectively. Saskatoon had the highest percentage $(39.5 \%)$ of participants with exposure to dusty job, and for the greatest number of years, 15.7 (details by sex are shown in e-Tables 1 and 2 ).

\section{Estimated Population Prevalence by Site}

The nine sampling cities are identified on a map of Canada, collectively representing where the majority of the Canadian population resides (Figure 1). Based on airflow limitation defined by the LLN ratio, the overall population prevalence of COPD was highest in Montreal, $14.7 \%$ (95\% CI, 11.4-18.0), and lowest in Quebec City, $8.3 \%$ (95\% CI, 5.8-10.8).

The estimated population prevalence for the presence of airflow limitation and its severity based on GOLD grades are shown for the nine study sites and separately for men and women in Table 2. In the overall estimated population prevalence, regardless of the definition (LLN or FR) used to define airflow limitation, the highest prevalence was in the same three locations: Montreal (LLN: 14.7\%, FR: 19.0\%), Kingston (LLN: 13.9\%, FR: 19.9\%) and Vancouver (LLN: 13.4\%, FR: 19.3\%).

Table 2 shows that the overall population prevalence of COPD in men was $18.3 \%(\mathrm{n}=428)$ by FR and $10.9 \%(\mathrm{n}=$ 241) by LLN ratio, while in women, the overall prevalence was $14.4 \%(n=413)$ and $11.4 \%(n=316)$, respectively. Overall, across all nine Canadian cities, the prevalence was $16.2 \%(95 \% \mathrm{CI}, 14.5-17.8)$ by FR and $11.2 \%$ (9.7-12.6) by LLN, with a difference of $5 \%$ between the two spirometric definitions. The majority of the population with airflow obstruction is classified as GOLD grade 1 $(8.6 \%)$ and grade $2(6.4 \%)$, indicating mild and moderate airflow limitation, respectively.

\section{Heterogeneity of Risk Factors by Site and by Sex}

The participants' characteristics of age, history of asthma, history of childhood hospitalization, smoking history, smoking status, and cumulative exposure in pack-years and their association with COPD as defined by airflow limitation below the LLN were summarized as odds ratio adjusted for potential confounding covariates in forest plots (Figures 2-7). The forest plots show: 1) risk association by site for men and women separately and the overall pooled estimate; 2) the site heterogeneity denoted by $\mathrm{I}^{2}$ and its significance on the overall risk association with COPD in the population. The overall adjusted odds ratio [aOR, 95\% CI] for age was $1.56(1.33,1.84)$ : $1.64(1.30,2.08)$ in men; 1.49 $(1.18,1.88)$ in women (Figure 2$)$. A history of physiciandiagnosed asthma (Figure 3) and childhood hospitalization for respiratory illness (Figure 4) were risk factors, with overall OR $3.30(2.42,4.49)$ and $1.81(1.17,2.80)$, respectively. In particular, men with a history of asthma were at an even higher risk for COPD, OR 4.28 (2.18, 8.37), compared to women, OR $2.86(1.98,4.14)$. With regards to smoking as a risk factor for COPD, our data compared several aspects, including ever vs never-smoking history, current vs former smoking status, and number of pack-years. Current smoking was associated with the highest odds ratio for COPD (OR 3.49; 95\% CI 2.55, 4.80) (Figure 5), followed by ever-smoking (OR 2.25; 95\% CI 1.81, 2.78) (Figure 6), and total pack-years smoked (OR 1.32; 95\% CI 


\begin{tabular}{|c|c|c|c|c|c|c|c|c|c|c|c|c|c|c|}
\hline 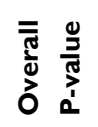 & & $\begin{array}{l}\overline{8} \\
\text { ¿ } \\
\text { v }\end{array}$ & $\begin{array}{l}\overline{8} \\
\text { ठ } \\
\text { v }\end{array}$ & 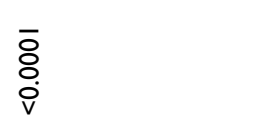 & $\begin{array}{l}\overline{8} \\
\text { ठ } \\
\text { v }\end{array}$ & $\begin{array}{l}\overline{8} \\
\text { o } \\
\text { v }\end{array}$ & $\begin{array}{l}\frac{n}{+0} \\
0 \\
0\end{array}$ & $\begin{array}{l}\overline{8} \\
\text { o } \\
\text { v }\end{array}$ & $\begin{array}{l}\overline{8} \\
\dot{0} \\
\mathrm{v}\end{array}$ & চ্口 & $\begin{array}{l}\text { oे } \\
\text { o } \\
0\end{array}$ & $\frac{\delta}{0}$ & $\begin{array}{l}\overline{8} \\
\text { o } \\
\text { v }\end{array}$ & $\begin{array}{l}\bar{\delta} \\
\text { ठ } \\
\text { v }\end{array}$ \\
\hline 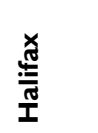 & $\stackrel{\infty}{\stackrel{\infty}{\sigma}}$ & 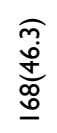 & 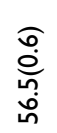 & 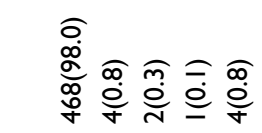 & $\frac{\widehat{n}}{\stackrel{0}{a}}$ & $\begin{array}{l}\widehat{T} \\
\text { o } \\
\text { in } \\
\underline{\underline{n}}\end{array}$ & 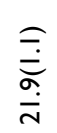 & 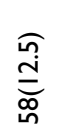 & 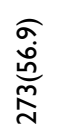 & 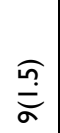 & 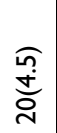 & 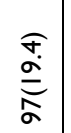 & 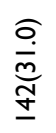 & 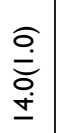 \\
\hline 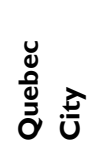 & 号 & 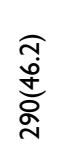 & 吕 & 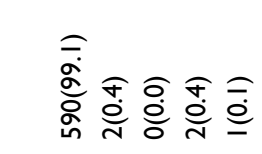 & 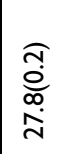 & $\begin{array}{l}\text { त̂. } \\
\stackrel{\infty}{\infty} \\
\stackrel{+}{\dot{x}}\end{array}$ & $\widehat{\overline{\bar{\Xi}}}$ & 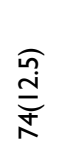 & 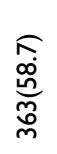 & 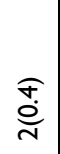 & 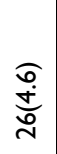 & 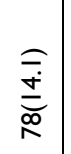 & $\frac{\substack{i\\
}}{\underline{d}}$ & 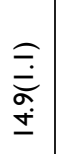 \\
\hline 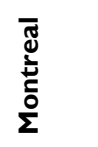 & 命 & 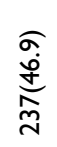 & 令 & 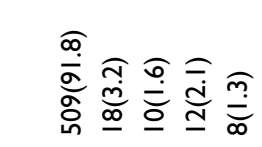 & 命 & 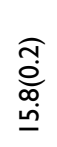 & 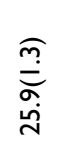 & 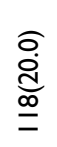 & 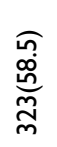 & 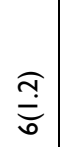 & 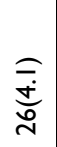 & $\underset{\substack{\sigma \\
\stackrel{\infty}{\infty}}}{\stackrel{\infty}{\underline{\infty}}}$ & 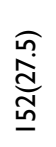 & 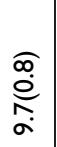 \\
\hline 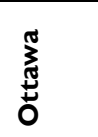 & $\stackrel{\dddot{o g}}{+}$ & 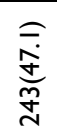 & 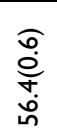 & 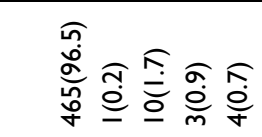 & 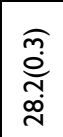 & $\begin{array}{l}\widehat{\text { }} \\
\stackrel{\rho}{m} \\
\underline{0}\end{array}$ & 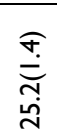 & $\widehat{\overline{\overline{\bar{\sigma}}}}$ & 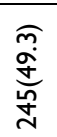 & 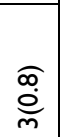 & 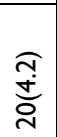 & ô. & 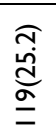 & 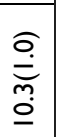 \\
\hline 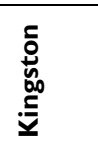 & 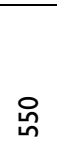 & 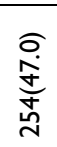 & $\underset{\substack{0 \\
\infty}}{\stackrel{0}{\infty}}$ & 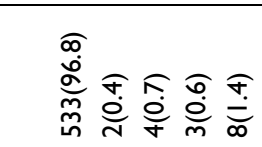 & $\begin{array}{l}\text { } \\
\stackrel{0}{0} \\
\substack{n \\
\infty}\end{array}$ & 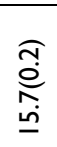 & 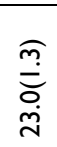 & $\stackrel{\widehat{\bar{m}}}{\stackrel{\hat{\sigma}}{\pi}}$ & 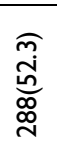 & $\underset{\stackrel{r}{e}}{\stackrel{c}{n}}$ & 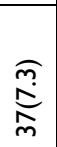 & 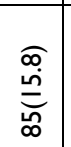 & 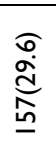 & 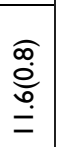 \\
\hline 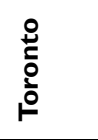 & $\stackrel{\infty}{\mathfrak{H}^{\prime}}$ & 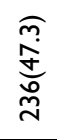 & 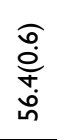 & 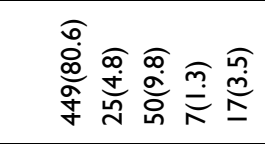 & तิ & 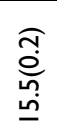 & 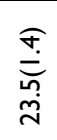 & 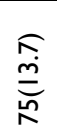 & 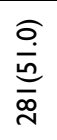 & 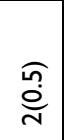 & 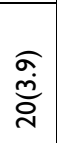 & $\underset{\substack{\infty \\
\stackrel{a}{\sigma}}}{\stackrel{a}{a}}$ & 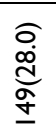 & 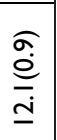 \\
\hline 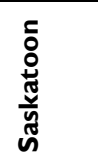 & i̊̀ & 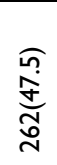 & 吕 & 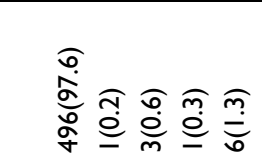 & 命 & 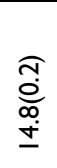 & 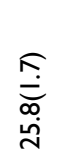 & 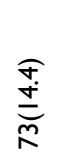 & 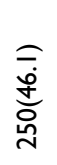 & $\frac{\sigma}{\stackrel{\sigma}{\Xi}}$ & 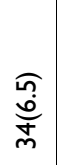 & 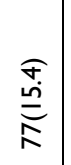 & 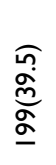 & 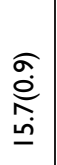 \\
\hline 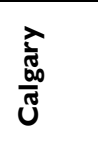 & ৪্ট & 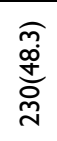 & 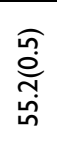 & 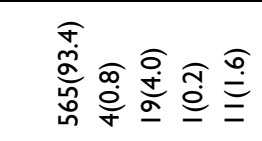 & $\begin{array}{c}\widehat{0} \\
\stackrel{0}{0} \\
\stackrel{\infty}{\infty} \\
\stackrel{N}{0}\end{array}$ & 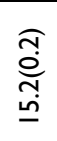 & $\widehat{\overline{\dot{\pi}}}$ & 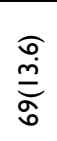 & 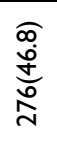 & 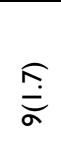 & $\widehat{\overline{\bar{c}}}$ & 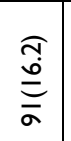 & 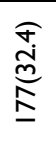 & 竞 \\
\hline 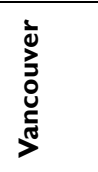 & ڤ̊ & 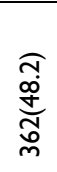 & 帒 & 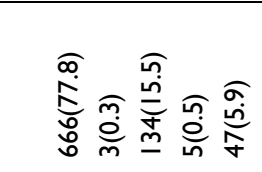 & 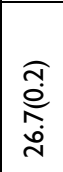 & $\begin{array}{c}\widehat{\bar{e}} \\
\text { ț } \\
\underline{\underline{u}}\end{array}$ & 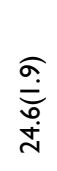 & $\stackrel{\substack{n \\
\stackrel{n}{N}}}{=}$ & 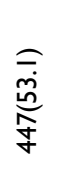 & & $\underset{\substack{a \\
\infty}}{\frac{\infty}{n}}$ & 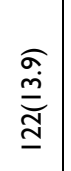 & 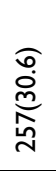 & 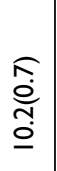 \\
\hline 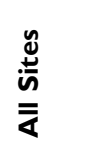 & $\frac{2}{n}$ & 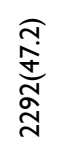 & 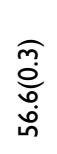 & 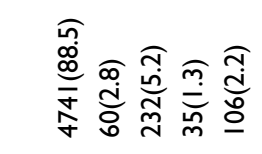 & $\frac{\widehat{\bar{i}}}{\stackrel{i}{\sim}}$ & $\begin{array}{l}\widehat{\overline{0}} \\
\underline{0} \\
\underline{\underline{0}}\end{array}$ & $\begin{array}{l}\text { Ẽ } \\
\stackrel{+}{d} \\
\dot{d}\end{array}$ & 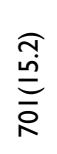 & 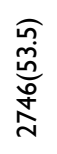 & 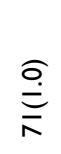 & 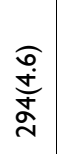 & 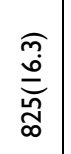 & 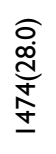 & 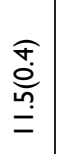 \\
\hline 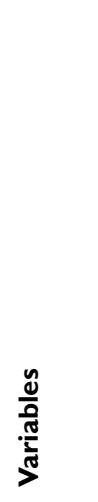 & 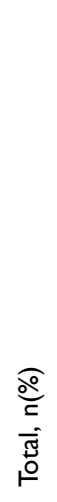 & 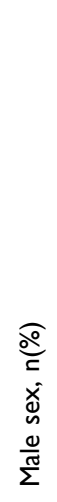 & 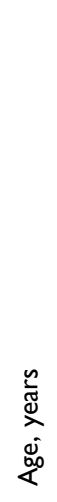 & 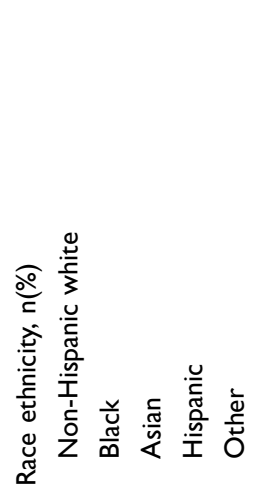 & 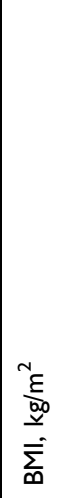 & 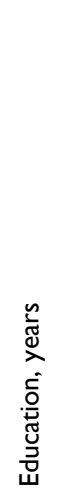 & 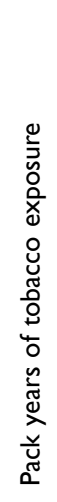 & 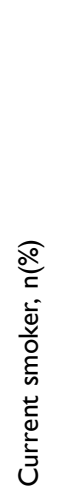 & 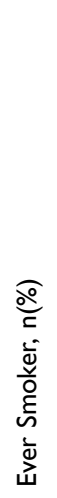 & 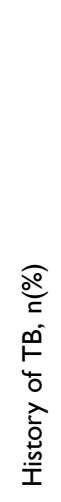 & 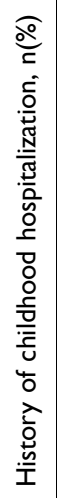 & 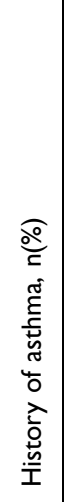 & 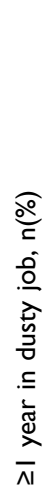 & 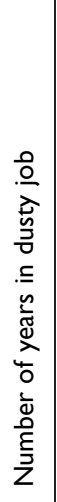 \\
\hline
\end{tabular}




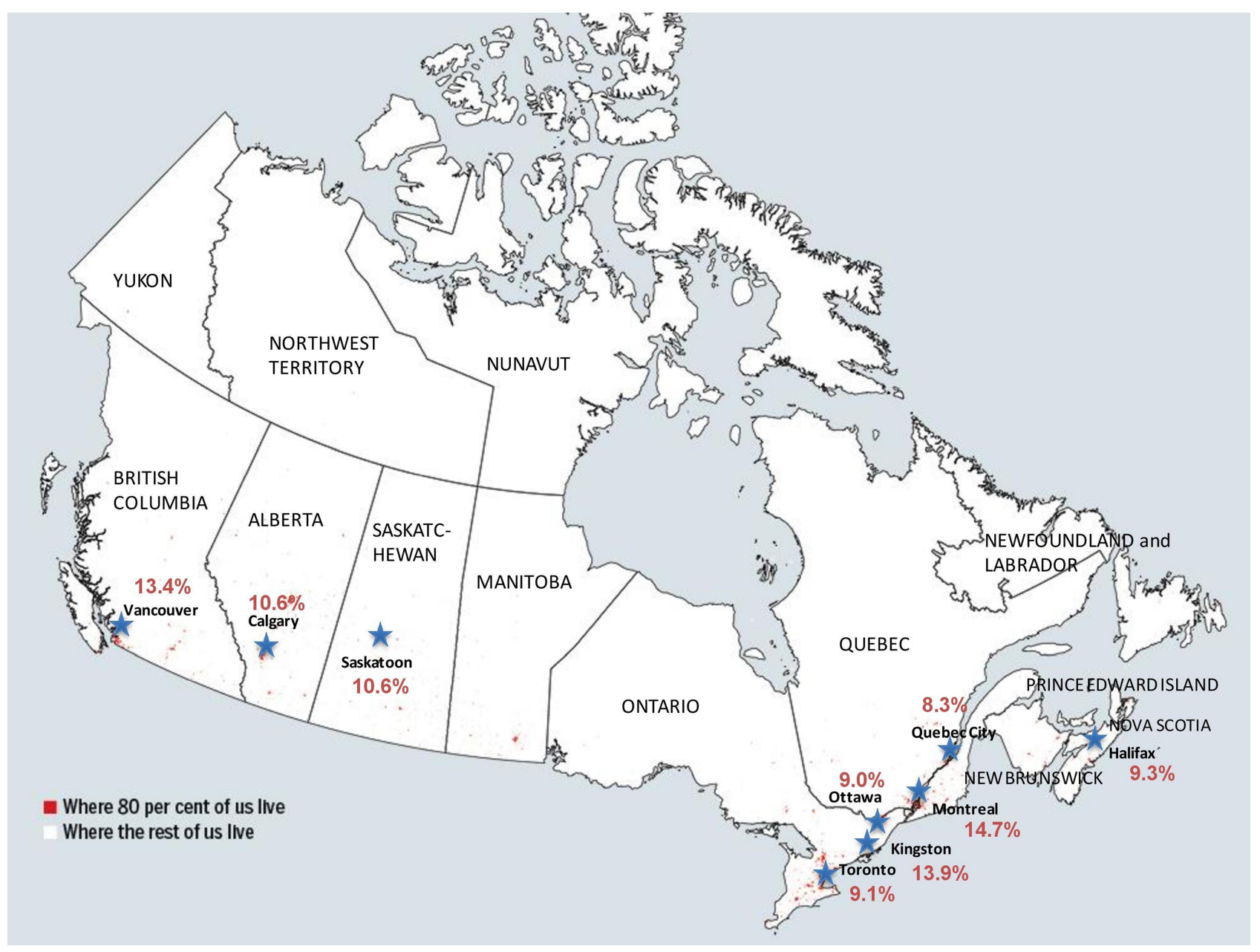

Figure I Geographical locations of the nine sampling urban cities in Canada. Overall population prevalence of COPD expressed as percentages. Airflow limitation was defined by FEVI/FVC <5th percentile (LLN). These sites collectively represent where the majority of the Canadian population resides.

1.22, 1.43) (Figure 7). There was significant heterogeneity among the nine sampling sites in age and pack-years of smoking history, I-squared $=40.8 \%, p=0.037$ and $48.5 \%$, $\mathrm{p}=0.011$, respectively, but not for the other risk factors.

The impact of these risk factors is further magnified with increasing spirometric severity as demonstrated by the higher odds ratio in moderate-severe COPD (Table 3), particularly in those with a history of asthma, aOR 5.22 $(3.54,7.70)$.

Table 4 shows the results of the additional heterogeneity analysis to test whether the variation in prevalence for COPD among sites is substantially reduced by adjustment for risk factors in this study. The results of Tau ${ }^{2}$ analyses further confirmed that there was significant heterogeneity among the nine sites without the influence of risk factors, with COPD as the outcome. When these risk factors were introduced into the model and $\mathrm{Tau}^{2}$ was adjusted for sex, age, smoking status, asthma, and childhood hospitalization, the variance decreased. The mean difference between the two analyses was significant, $0.023(0.001,0.037)$; $p<$ 0.001 . The variation among sites is therefore explained by differences in risk factors.

\section{Discussion}

The present study reported population prevalence for COPD in Canada using two spirometric criteria and provided new data on the heterogeneity of prevalence across the country. We found heterogeneity in age and pack-years of smoking (reflecting exposure burden) across sites, while a history of asthma, childhood hospitalization, and smoking patterns were homogeneous across sites in Canada; and that the variation in prevalence across sites can be reduced by adjustment for these risk factors. We have also shown that the risk factors associated with COPD are qualitatively similar across all grades of severity of COPD but the strength of association increased with 


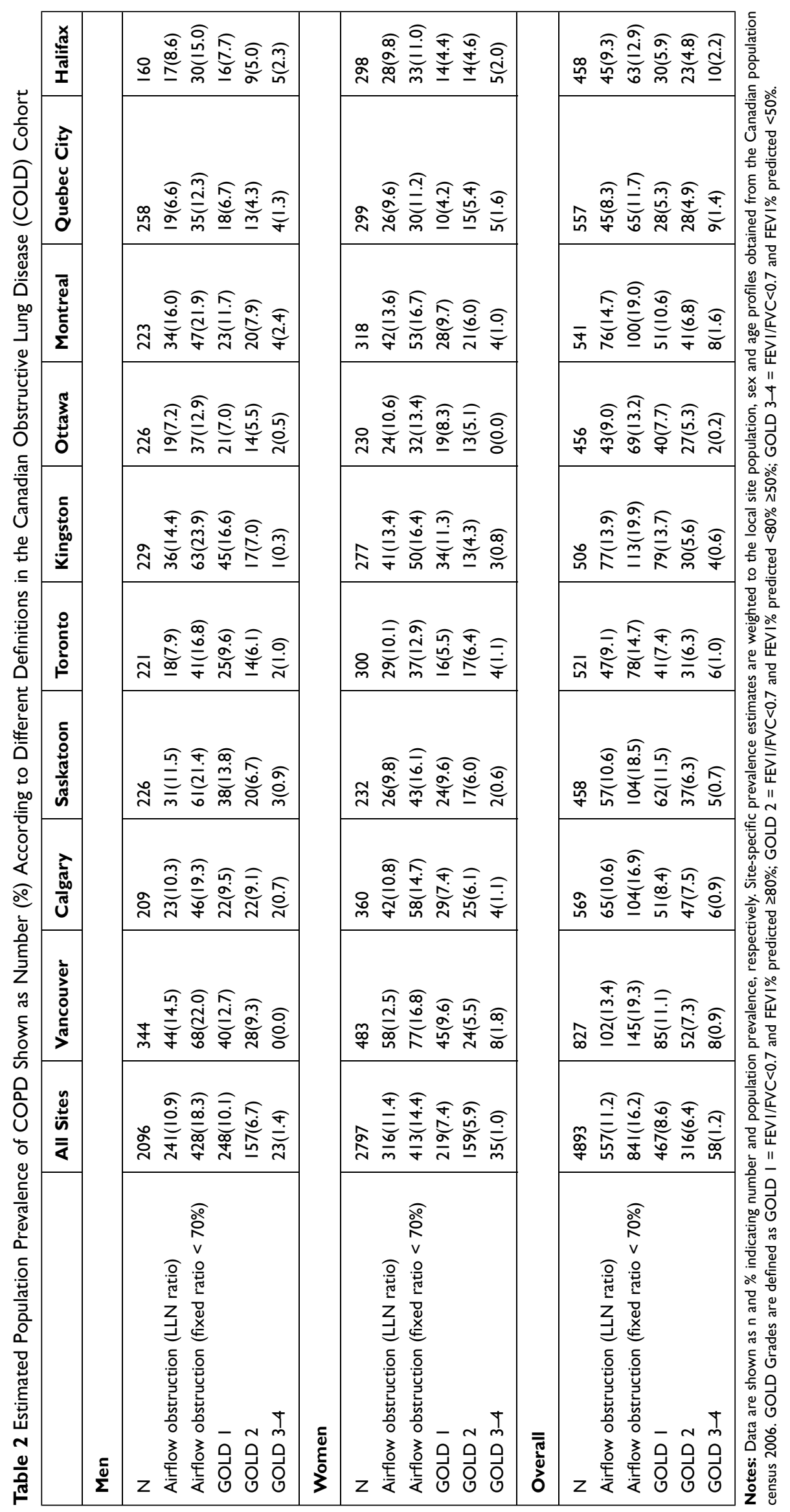




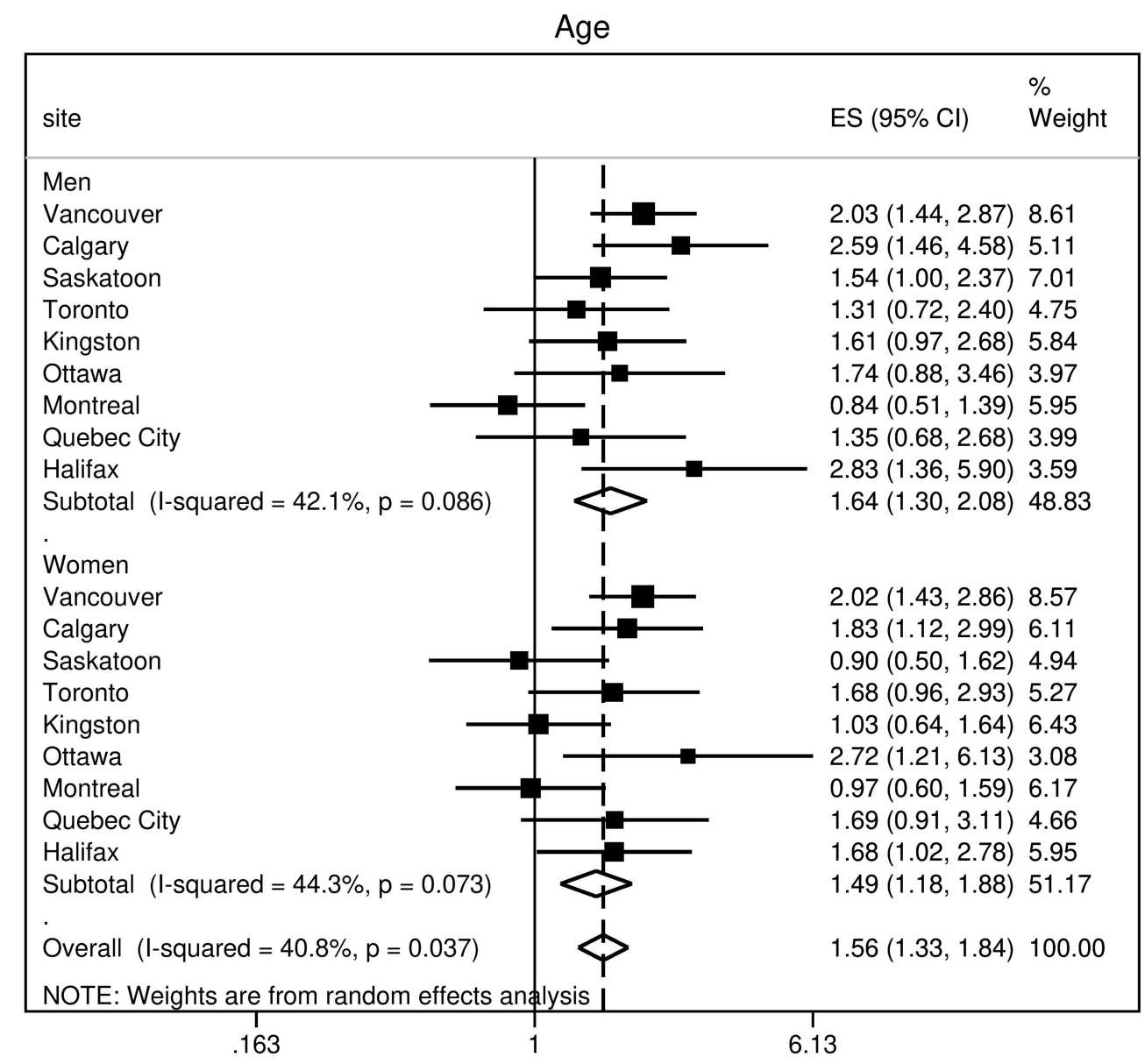

Figure 2 Age as a risk factor for COPD, showing significant heterogeneity in risk among the nine sampling sites. Results are shown for men and women and for the whole cohort. The $\mathrm{x}$-axis is the log-scaled, but the values labelled under the line are the actual odds ratio. COPD was defined by airflow limitation (FEVI/FVC < LLN); ES ( $95 \%$ Cl) is the adjusted odds ratio and $95 \%$ confidence interval, aOR $(95 \% \mathrm{Cl})$, for every 10 -year increase in age, adjusted for school years, pack-years, asthma, childhood hospitalization, and dusty job exposure.

severity, suggesting that a higher risk could be linked to the development of severe disease. Taken together, accurate population data on spirometry-based COPD prevalence and a better understanding of the heterogeneity of major risk factors in different regions of the country can help inform public policymakers on target strategies in reducing COPD and its severity in the country.

\section{COPD Prevalence in Canada}

Determination of spirometry-based population prevalence is crucial to understanding the impact of COPD in Canada. Previous reports on the prevalence of COPD in Canada were largely based on surveys with self-reported history of chronic bronchitis or emphysema, ${ }^{20}$ administrative databased criteria in single or multiple centers, ${ }^{21,22}$ or criteria that only relied on pre-bronchodilator spirometry. ${ }^{23}$ Following the methodology in the BOLD study, ${ }^{9}$ the use of random sampling and a standardized protocol, including the definition of COPD by strict post-bronchodilator criteria, has allowed for more accurate determination of prevalence. Although some data on COPD prevalence on five Canadian sites were previously published, ${ }^{6}$ this study adds to the literature by providing robust and extensive data on the Canadian population prevalence of COPD documented by the current two widely used clinical measures of postbronchodilator $\mathrm{FEV}_{1} / \mathrm{FVC}$ ratio $(\mathrm{FR}<0.70$ and $\mathrm{LLN}<5$ th percentile). Our study emphasizes the importance of the spirometric definition when quoting prevalence of COPD and showed the impact on the interpretation on sex difference in prevalence.

An interesting observation is that more than half of the overall COPD cases were classified as mild (GOLD 1, $\mathrm{FEV}_{1} / \mathrm{FVC}<0.7$ and $\mathrm{FEV}_{1 \%}$ predicted $>80 \%$ ) in the population. This pattern is expected from a population-based sample of non-institutionalized men and women. Yet, it is pertinent to health-care planning as this group though 


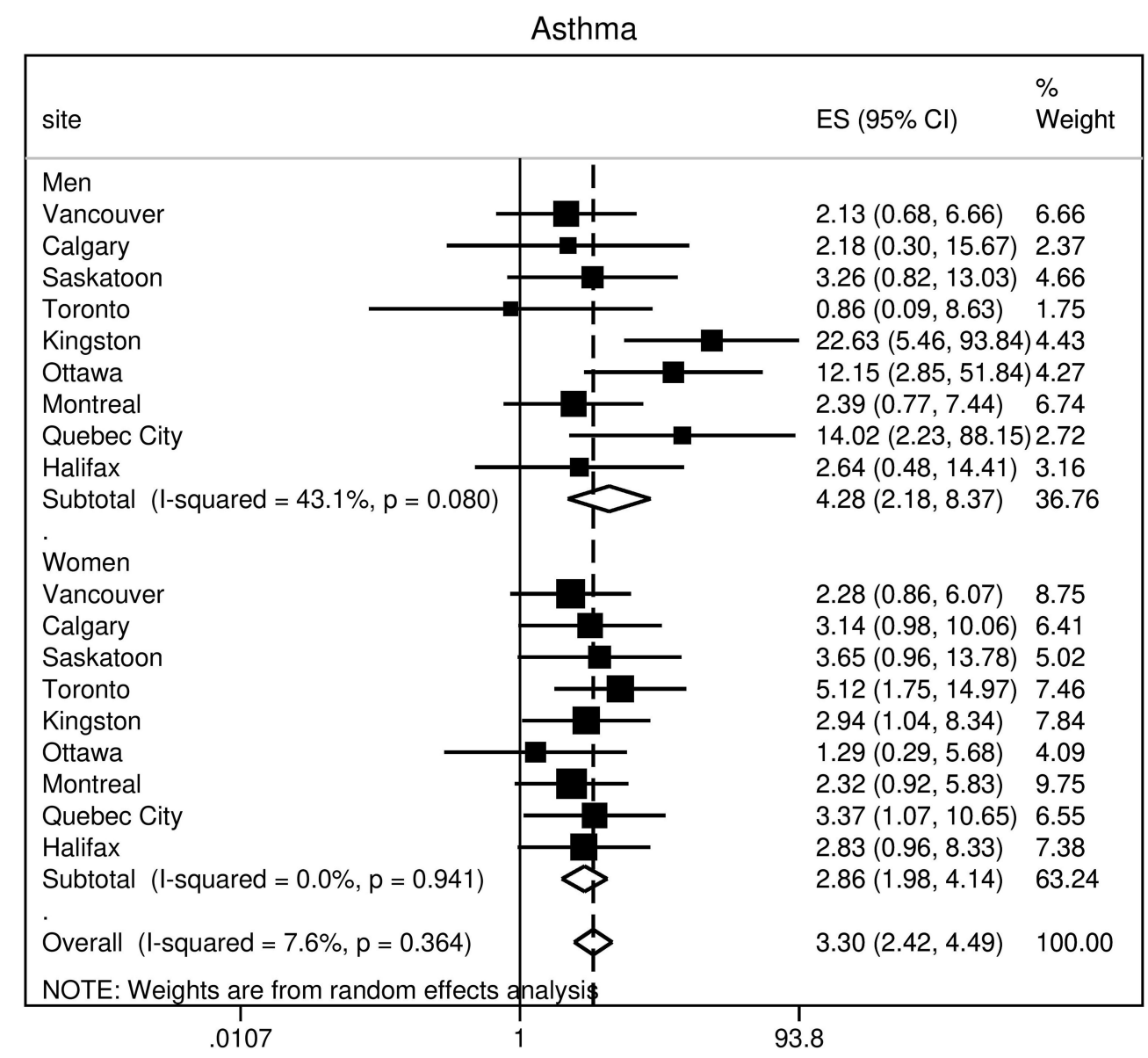

Figure 3 Asthma as a risk factor for COPD across nine sites. Results are shown for men and women and for the whole cohort. COPD was defined by airflow limitation (FEVI/FVC < LLN); ES $(95 \% \mathrm{Cl})$ is the adjusted odds ratio and $95 \%$ confidence interval, aOR $(95 \% \mathrm{Cl})$, adjusted for age, school years, pack-years, and dusty job exposure. There is no site heterogeneity demonstrated.

"mild" can still experience exacerbations and utilize health-care resources with a significant economic and social impact. ${ }^{24}$ These patients should be provided education on COPD, and be encouraged on lifestyle optimization and smoking cessation to prevent disease progression. Early intervention is essential as our findings have shown that the impact of smoking-related risk factors is further magnified in more severe disease.

The Canadian prevalence of GOLD grade 2 to 4 COPD $\left(\mathrm{FEV}_{1} / \mathrm{FVC}<0.7\right.$ and $\mathrm{FEV}_{1 \%}$ predicted $\left.<80 \%\right)$ is lower (7.5, $\mathrm{SD} 0.6)$ compared to that reported in the BOLD study (10.1\%, SE 4.8). ${ }^{9}$ In the PLATINO study, the prevalence of COPD varied significantly among the five Latin American locations, ranging from $7.8 \%$ (95\% CI, 5.9-9.7) in Mexico City to almost 20\% (95\% CI, 17.2-22.2) in Montevideo. ${ }^{11}$ In a systematic review that did not specifically use spirometry to define
COPD, the global pooled prevalence of COPD was $9.9 \%(95 \% \mathrm{CI}, 8.2-11.8)$ in the population over 40 years old. ${ }^{25}$

\section{Heterogeneity of Risk Factors}

Our study indicates that the burden of main risk factors underlying the development of COPD differs among Canadian cities. It is conceivable that the heterogeneity of COPD prevalence within Canada may also result from other unique factors not included in this analysis. For example, geographic variation including altitude and temperature has also been proposed as explanation for differences in COPD prevalence in other countries. ${ }^{11,26}$ Although sex, age, and smoking contribute to two-thirds of the variability in COPD prevalence, a significant portion of the variability remains unexplained. ${ }^{26}$ 


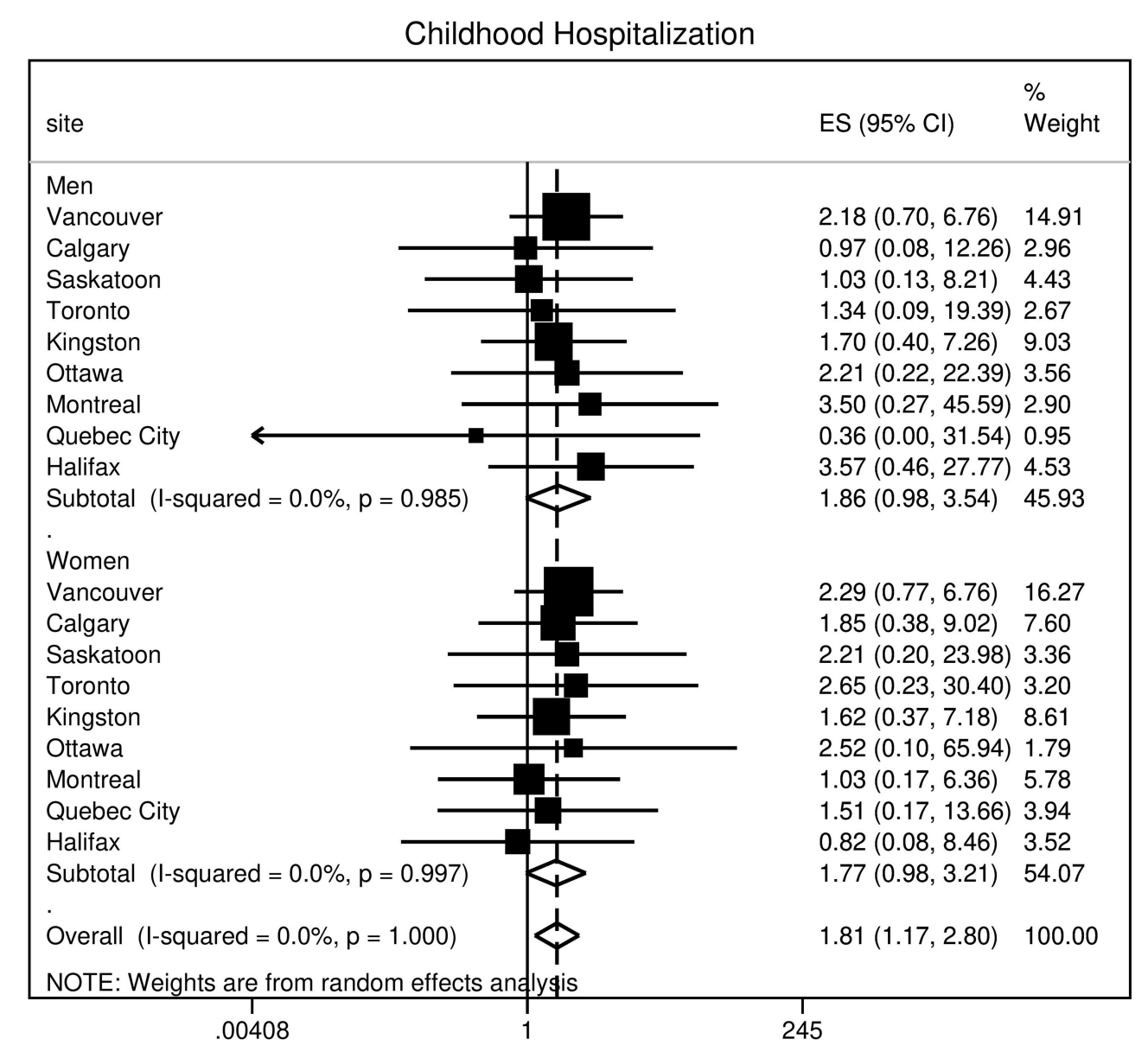

Figure 4 Childhood hospitalization as a risk factor for COPD across nine sites. Results are shown for men and women and for the whole cohort. COPD was defined by airflow limitation (FEVI/FVC < LLN); ES ( $95 \% \mathrm{Cl}$ ) is the adjusted odds ratio and $95 \%$ confidence interval, aOR $(95 \% \mathrm{Cl})$, adjusted for age, school years, pack-years, and dusty job exposure There is no site heterogeneity demonstrated.

Our study found that a history of asthma or childhood hospitalization for respiratory illnesses is just as important as smoking in the development of spirometrically confirmed COPD. While long-term cigarette smoking behaviour is the most important and well-studied cause of COPD in the Western World, ${ }^{27-29}$ COPD can occur in never-smokers which suggests the importance of other risk factors in determining an individual's susceptibility to $\mathrm{COPD} .^{2,30}$ In never-smokers, occupational exposures including organic and inorganic dusts, chemical agents, and fumes are related to the development of COPD. In patients with established COPD, these exposures can worsen the morbidity, quality of life, and exacerbation risk. ${ }^{31,32}$ Medical events during gestation and birth, and exposures during childhood and adolescence affect lung growth and subsequent function in adult life. ${ }^{33,34} \mathrm{~A}$ history of severe childhood respiratory infection and hospitalization is associated with reduced lung function and increased respiratory symptoms in adulthood. ${ }^{35,36}$ In a prospective observational study over 20 years, subjects with physicianconfirmed active asthma were identified as a risk factor for COPD (HR 12.5, 95\% CI 6.83-22.8), chronic bronchitis (HR 10.0, 4.90-20.2), and emphysema (HR 17.0, $8.30-34.8) .{ }^{37}$ It is intriguing that the risk burden of asthma and childhood hospitalization for COPD, unlike the risk burden of exposure to cigarette smoke, did not show regional variation in Canada.

\section{Gender Differences}

Recent research on gender-specific prevalence suggested that the prevalence has been rising more rapidly in females compared to males. ${ }^{38,39}$ The discordance in the sex difference in COPD prevalence defined by the two definitions of fixed ratio and lower limits of normal ratio cut-offs is intriguing. We think this is explained by the fact that men have larger absolute lung volumes than women (ie increased FVC); thus, it is easier to develop a reduced FEV1/FVC ratio with progressive airflow impairment. 


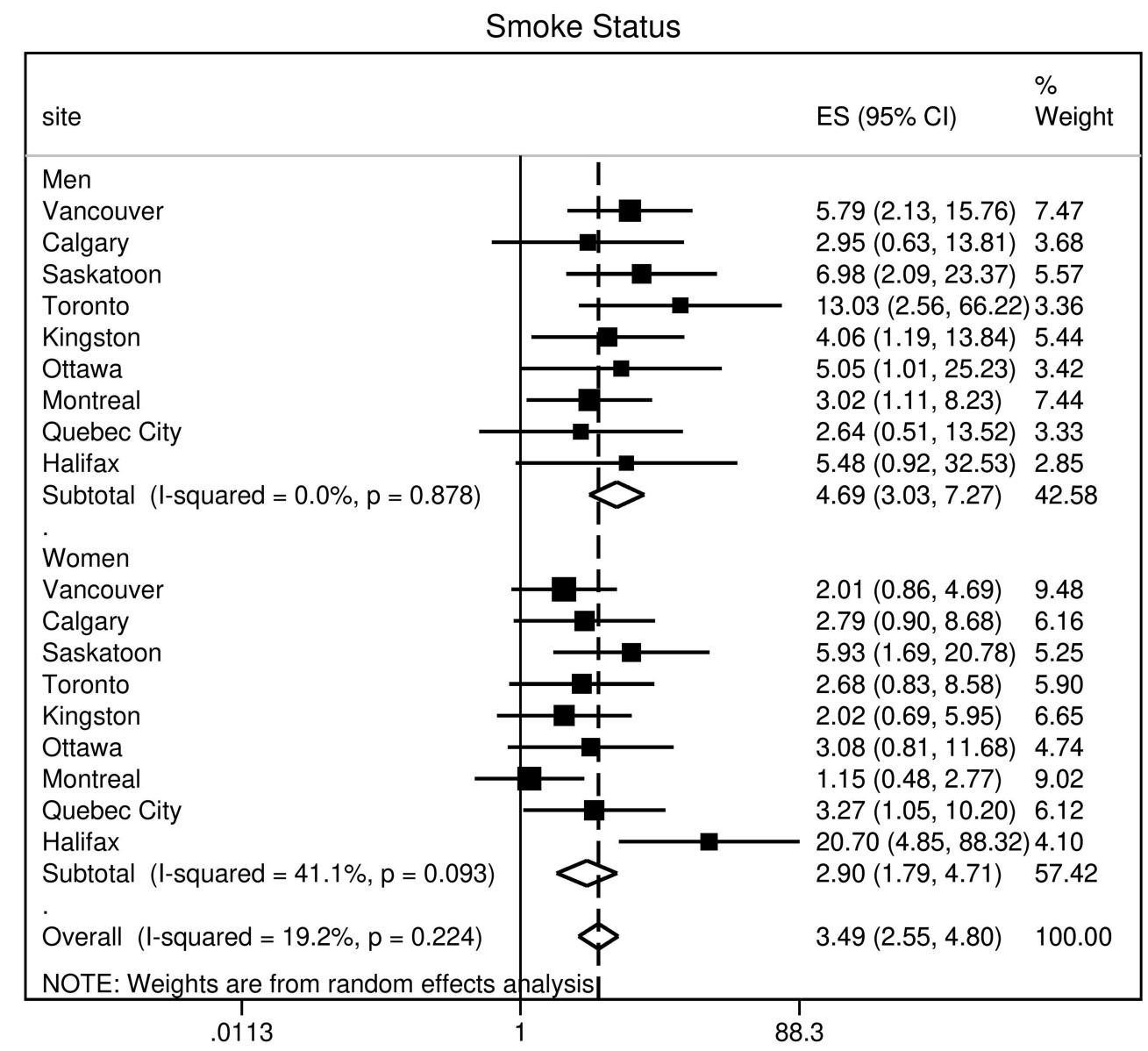

Figure 5 Current vs former smoking as a risk factor for COPD across nine sites. Results are shown for men and women and for the whole cohort. COPD was defined by airflow limitation (FEVI/FVC < LLN); ES ( $95 \% \mathrm{Cl}$ ) is the adjusted odds ratio and $95 \%$ confidence interval, aOR $(95 \% \mathrm{Cl})$, adjusted for age, school years, asthma, childhood hospitalization, and dusty job exposure. There is no significant site heterogeneity demonstrated.

This issue can be largely mitigated when the FEV1/FVC ratio is adjusted for age, sex and ethnicity and presented as $\%$ of predicted value. ${ }^{40}$

The phenomenon of higher prevalence for men when using fixed ratio and lower or almost similar sex prevalence when using LLN has been shown previously in a paper from the Netherlands where the use of LLN for stage 1 [FEV $1 / \mathrm{FVC}<\mathrm{LLN}$ and FEV1 $\geq 80 \%$ pred] at least nearly fully abolishes the sex-related differences in the prevalence of COPD which is observed with the fixed ratio. ${ }^{40}$ Hence, our observation is consistent with these previous findings and further supports the recommended use of LLN threshold rather than fixed ratio threshold for defining COPD as set out in the guidelines of the ATS/ERS. ${ }^{12}$ Furthermore, the analyses of data from the BOLD initiative which used methodology identical to this COLD study had clearly shown that the use of the FEV1/FVC $<$ LLN criterion instead of the FEV1/FVC $<0.7$ minimized known age biases and better reflect clinically significant irreversible airflow limitation. ${ }^{18}$

Finally, our finding that significant site heterogeneity in the risk for COPD due to cumulative tobacco exposure (pack-years) was seen in men but not in women suggests that the site relative proportion of men and women could also be an explanation for some of the variation in prevalence, consistent with our previous observation that site variation in prevalence appeared to be driven by age and sex. $^{6}$

\section{Limitations}

There are several limitations of this study. The presented data may not necessarily reflect rural settings and certain ethnic groups in Canada. For example, the Aboriginal population of Canada has a higher incidence and burden of disease compared to non-Aboriginal Canadian population, likely as a result of disparities in risk factors including smoking. ${ }^{41}$ A previous systematic 


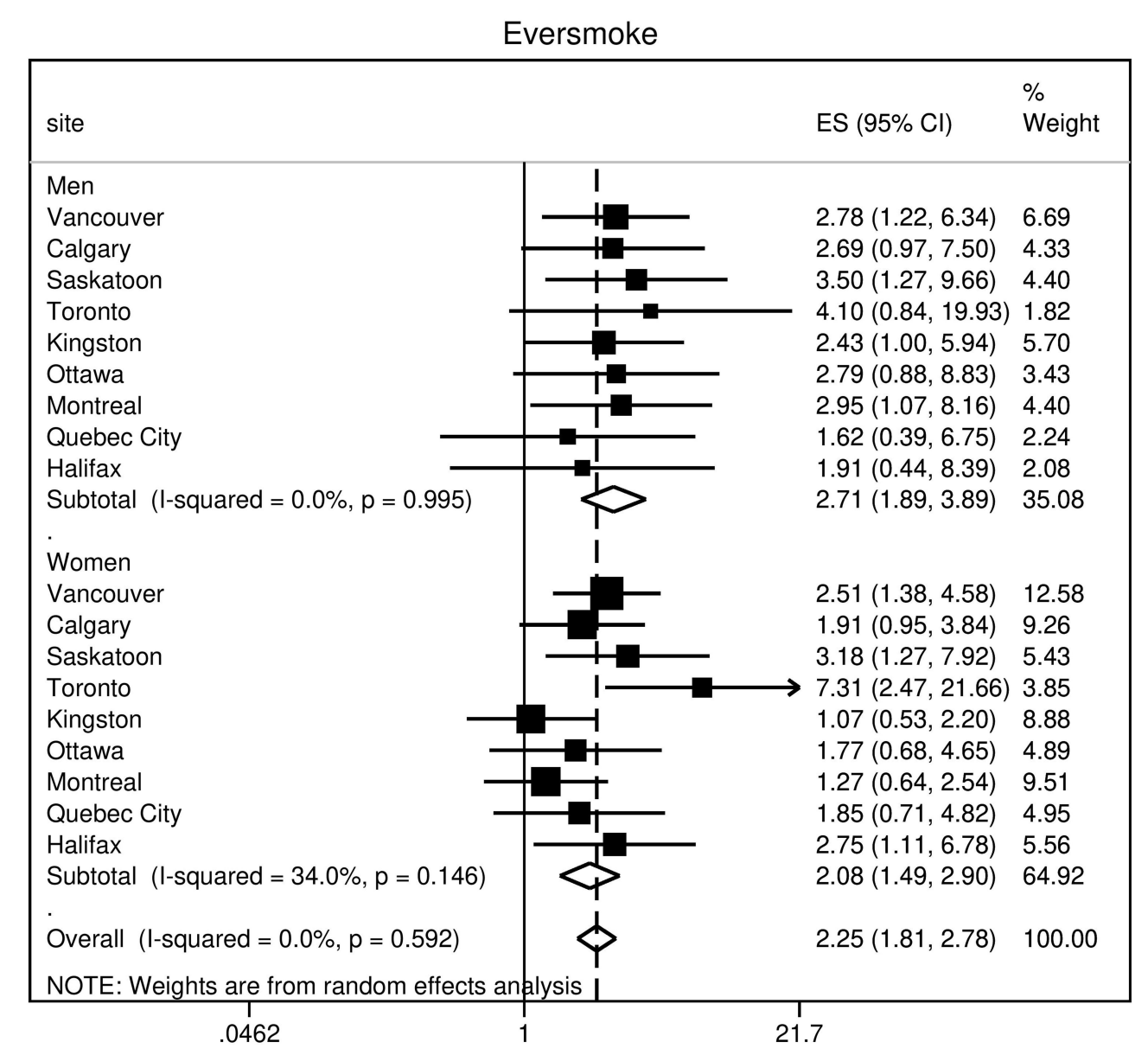

Figure 6 Ever-smoking as a risk factor for COPD across nine sites. Results are shown for men and women and for the whole cohort. COPD was defined by airflow limitation (FEVI/FVC < LLN); ES $(95 \% \mathrm{Cl})$ is the adjusted odds ratio and $95 \%$ confidence interval, aOR $(95 \% \mathrm{Cl})$, adjusted for age, school years, asthma, childhood hospitalization, and dusty job exposure. There is no significant site heterogeneity demonstrated.

review demonstrated that COPD prevalence is generally lower in rural settings. ${ }^{42}$ Nonetheless, our study obtained samples from cities where the collective majority of the Canadian population resided. The delay in reporting these data, which were collected more than 10 years ago, is a limitation due to the potential influence of changes in age profile and ethnic distribution of the population over the past decade. Comparing the Canadian Census data in 2006 and 2016, the proportion of individuals over age 40 has increased only slightly by $2.3 \%$ (e-Figure 2). The ethnicity of the Canadian population has shifted towards an increase in non-white group by $7.6 \%$ between 2006 and 2016 (e-Figure 2). Visible minority Canadians were underrepresented in this study. The gap in health data and research on visible minorities in Canada has been previously documented $^{43}$ and may be due to various barriers to participation. Finally, some subjects with airflow limitation may have incompletely reversible asthma rather than COPD in this study based on spirometrically defined COPD.

\section{Conclusions}

Our study has confirmed that COPD remains a significant health issue in Canada, as shown by the population prevalence based on post-bronchodilator spirometry and defined by two globally popular definitions, thus, providing representative and reliable population estimates of this disease. Sex difference in prevalence is influenced by the choice of spirometric definition for COPD. Significant site heterogeneity in risk factor was demonstrated in the burden of tobacco exposure (increasing pack-years) rather than the pattern of smoking and that the variation in prevalence across sites can be reduced by adjustment for risk factors. The strength of association between COPD and the analysed 


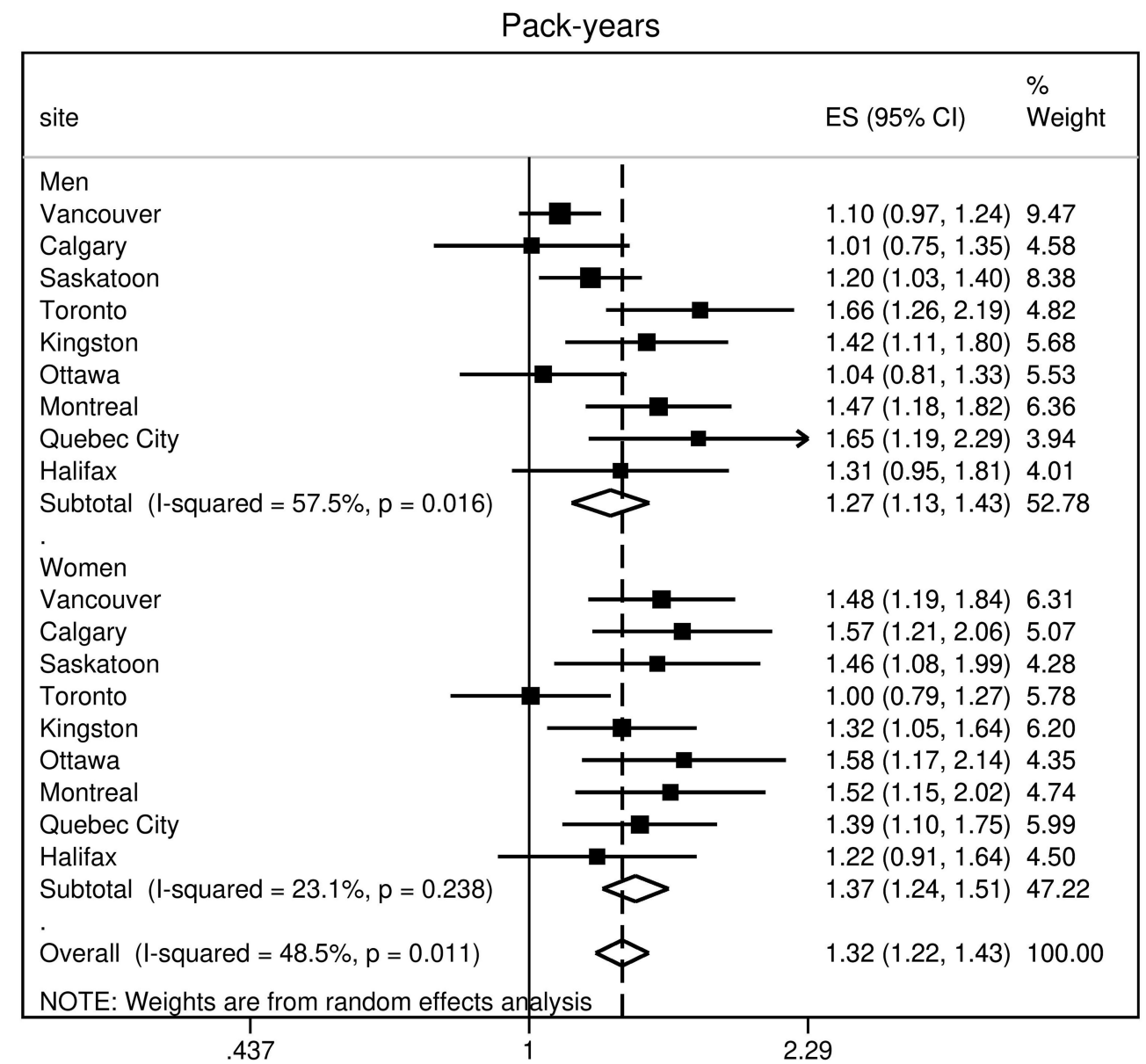

Figure 7 Smoking in pack-years as a risk factor for COPD, showing significant site heterogeneity in risk among the nine sampling sites. Results are shown for men and women and for the whole cohort. COPD was defined by airflow limitation (FEVI/FVC < LLN); ES ( $95 \% \mathrm{Cl}$ ) is the adjusted odds ratio and $95 \%$ confidence interval, aOR ( $95 \%$ $\mathrm{Cl}$ ) for every 10-pack-year increase, adjusted for age, school years, asthma, childhood hospitalization, and dusty job exposure.

risk factors was magnified with disease severity. The study emphasizes the importance of understanding regional heterogeneity of risk factors for $\mathrm{COPD}$, as this knowledge is essential for interpretation of the prevalence of COPD and help inform health policy planning for COPD within a country. More studies are needed to better understand the link between variations in risk factors and COPD.

Table 3 Overall Adjusted Odds Ratio (aOR) for the Risk Factors for COPD of Different Severity: Mild (GOLD Grade I) and Moderate-Severe (GOLD Grade 2-4)

\begin{tabular}{|l|l|l|}
\hline & $\begin{array}{l}\text { Mild COPD (GOLD Grade I) } \\
\text { aOR }\end{array}$ & $\begin{array}{l}\text { Moderate-to-Severe COPD (GOLD Grade 2-4) } \\
\text { aOR }\end{array}$ \\
\hline Age, years & $1.99(1.80,2.20)$ & $2.22(1.87,2.63)^{*}$ \\
Asthma (Y vs N) & $2.69(2.01,3.59)$ & $5.22(3.54,7.70)^{*}$ \\
Childhood hospitalization (Y vs N) & $1.89(1.21,2.94)$ & $3.07(2.02,4.66)$ \\
Ever-smoker status (Y vs N) & $1.65(1.33,2.05)$ & $3.05(2.24,4.16)$ \\
Lifetime tobacco exposure, pack-years $\#$ & $1.18(1.10,1.28)$ & $1.36(1.25,1.48)^{*}$ \\
Smoking status (current vs former) & $2.34(1.67,3.29)$ & $4.09(2.78,6.01)$ \\
\hline
\end{tabular}

Notes: GOLD Grades are defined as GOLD I = FEVI/FVC $<0.7$ and FEVI\% predicted $\geq 80 \%$; GOLD $2=$ FEVI/FVC $<0.7$ and FEVI\% predicted $\geq 50 \%$ and $<80 \%$; GOLD 3 $=\mathrm{FEVI} / \mathrm{FVC}<0.7$ and $\mathrm{FEVI} \%$ predicted $\geq 30 \%$ and $<50 \%$; GOLD $4=\mathrm{FEVI} / \mathrm{FVC}<0.7$ and FEVI\% predicted $<30 \%$. *Indicates presence of significant site heterogeneity. ${ }^{\#} \mathrm{Pack}$ years $=($ number of cigarettes per day/20) $\times$ number of years smoked. Separate models were constructed for each risk factor. Age adjusted for school years and eversmoking history. Asthma adjusted for age, school years, and ever-smoking history. Childhood hospitalization adjusted for age, school years, and ever-smoking history. Eversmoker status adjusted for age and school years. Lifetime tobacco exposure adjusted for age and school years. Smoking status adjusted for age and school years. Abbreviation: aOR, overall adjusted odds ratio for men and women. 
Table 4 Heterogeneity $\left(\mathrm{Tau}^{2}\right)$ for the Prevalence of COPD (LLN) Among the Nine Sites

\begin{tabular}{|l|l|l|l|l|}
\hline & Tau $^{2}$ without Risk Factors & $*$ Tau $^{2}$ with Risk Factors & ${ }^{*}$ Mean Difference (95\% Cl) & p-value \\
\hline Prevalence of COPD (LLN) & 0.053 & 0.030 & $0.023(0.001,0.037)$ & $<0.001^{\$}$ \\
\hline
\end{tabular}

Notes: ${ }^{*} \mathrm{Tau}^{2}$ is adjusted for sex, age, smoking status, asthma, and childhood hospitalization; ${ }^{\#}$ Mean difference for Tau ${ }^{2}(95 \% \mathrm{Cl})$ with and without risk factors; ${ }^{\$}$ Indicates a significant difference of $\mathrm{Tau}^{2}$ with and without risk factors.

\section{Abbreviations}

BOLD, International Burden of Obstructive Lung Disease; COPD, chronic obstructive pulmonary disease; COLD, Canadian Obstructive Lung Disease; FEV1, forced expiratory volume in 1 second; FR, fixed ratio; GOLD, Global Initiative for Chronic Obstructive Lung Disease; LLN, lower limit of normal.

\section{Acknowledgments}

The authors would also like to thank the men and women who participated in the study and individuals in the * CanCOLD Collaborative research Group: Jonathon Samet (the Keck School of Medicine of USC, California, USA); William M Vollmer (The Kaiser Permanente Center for Health Research in Portland, OR); Milo Puhan (John Hopkins School of Public Health, Baltimore, USA); Qutayba Hamid, Carolyn Baglole, Benjamin M Smith, Palmina Mancino, PeiZhi Li, Zhi Song, Dennis Jensen (McGill University, Montreal, QC, Canada); Yvan Fortier (Sherbrooke University, Sherbrooke, QC, Canada); Patricia McClean, Jane Duke, Andrea S Gershon, Teresa Toh (University of Toronto, Toronto, ON Canada); Mohsen Sadatsafavi (University of British Columbia, Vancouver, BC); Christine Lo, Sarah Cheng, Elena Un, Michael Cheng, Cynthia Fung, Faize Faroon, Olga Radivojevic, Sally Chung, Carl Zou, Rena Choi, Joe Comeau, Harvey Coxson, Miranda Kirby, Jonathon Leipsic, Cameron Hague (UBC James Hogg Research Center, Vancouver, BC, Canada); Curtis Dumonceaux (University of Calgary, Calgary, AB, Canada); Scott Fulton (Dalhousie University, Halifax, NS, Canada); Kathy Vandemheen (University of Ottawa, Ottawa, ON, Canada); Matthew McNeil, Kate Whelan (Queen's University, Kingston, ON, Canada); Cynthia Brouillard (University of Laval, Quebec City, QC, Canada); Ron Clemens, Janet Baran (University of Saskatchewan, Saskatoon, SK, Canada).

\section{Author Contributions}

All authors made substantial contributions to conception and design, acquisition of data, or analysis and interpretation of data; took part in drafting the article or revising it critically for important intellectual content; agreed to submit to the current journal; gave final approval of the version to be published; and agree to be accountable for all aspects of the work.

\section{Funding}

The Canadian Cohort Obstructive Lung Disease (CanCOLD; NCT00920348) study is currently funded by the Canadian Respiratory Research Network and the industry partners AstraZeneca Canada Ltd, Boehringer Ingelheim Canada Ltd, GlaxoSmithKline Canada Ltd, and Novartis. Researchers at RI-McGill University Health Centre Montreal and iCAPTURE Centre Vancouver lead the project. Previous funding partners were the Canadian Institutes of Health Research (CIHR; CIHR/Rx\&D Collaborative Research Program Operating Grants - 93326), the Respiratory Health Network of the Fonds de la recherche en santé du Québec (FRQS), and industry partners: Almirall; Merck Nycomed; Pfizer Canada Ltd; and Theratechnologies. The funding sponsors had no role in the study design; in the collection, analysis, and interpretation of data, in the writing of this manuscript or in the decision to submit this manuscript for publication. The funders had no role in the study design, data collection and analysis, or preparation of the manuscript.

\section{Disclosure}

Jean Bourbeau reports grants from CIHR, grants from Foundation of the MUHC, personal fees from Canadian Thoracic Society, personal fees from AstraZeneca, consultant/lecture for CHEST, advisor/lecture for Boehringer Ingelheim, Grifols, GlaxoSmithKline, and Novartis, lecture for Trudell, grants from Aerocrine, outside the submitted work.

François Maltais reports grants from CanCOLD, during the conduct of the study; grants from GlaxoSmithKline, AstraZeneca, Sanofi, personal fees from GlaxoSmithKline, Boehringer Ingelheim, Grifols, Novartis, unrestricted grants paid to their institution from Novartis, Boehringer Ingelheim, Grifols, and financial participation in Oxynov, a company 
which is developing an oxygen delivery system, outside the submitted work.

Darcy D Marciniuk reports grants from McGill University, during the conduct of the study; grants from AstraZeneca, Boehringer-Ingelheim, GlaxoSmithKline, Canadian Health Infowayfor Healthcare Improvement, Lung Association of Saskatchewan, and Canadian Institute of Health Research, Grifols, Novartis, Sanofi, Saskatchewan Health Research Foundation, Schering-Plough, and is an employee of the University of Saskatchewan, outside the submitted work.

Paul Hernandez reports personal fees from AstraZeneca, grants from Boehringer Ingelheim, Grifols, and Vertex, fees for medical advisory board from Actelion, GlaxoSmithKline, and Novartis, outside the submitted work.

Wan C Tan reports grants from Canadian Institute of Heath Research, rgrants from GlaxoSmithKline Canada Ltd, nothing from AstraZeneca Canada Ltd., nothing from Boehringer-Ingelheim Canada Ltd, nothing from Novartis, nothing from Merck, nothing from Pfizer, Canada, and nothing from Nycomed, Canada, during the conduct of the study; personal fees from GlaxoSmithKline, Canada and AstraZeneca, Canada, outside the submitted work.

The abstract of this paper was presented at the American Thoracic Society 2020 International Conference as a poster presentation with interim findings. The poster's abstract was published in "Poster Abstracts" in the American Journal of Respiratory and Critical Care Medicine: https://doi.org/10. 1164/ajrccm-conference.2020.201.1_MeetingAbstracts. A4566. This study complied with the Declaration of Helsinki.

\section{References}

1. DiBonaventura M, daCosta PR, Su J, et al. The burden of chronic obstructive pulmonary disease among employed adults. Int J Chron Obstruct Pulmon Dis. 2012;7:211-219. doi:10.2147/COPD.S29280

2. Chapman KR, Mannino DM, Soriano JB, et al. Epidemiology and costs of chronic obstructive pulmonary disease. Eur Respir J. 2006;27(1):188-207. doi:10.1183/09031936.06.00024505

3. López-Campos JL, Tan W, Soriano JB. Global burden of COPD: global burden of COPD. Respirology. 2016;21(1):14-23. doi:10.1111/resp.12660

4. Chapman KR, Bourbeau J, Rance L. The burden of COPD in Canada: results from the confronting COPD survey. Respir Med. 2003;97:S23S31. doi:10.1016/S0954-6111(03)80022-7

5. Rabe KF, Hurd S, Anzueto A, et al. Global strategy for the diagnosis, management, and prevention of chronic obstructive pulmonary disease: GOLD executive summary. Am J Respir Crit Care Med. 2007;176(6):532-555. doi:10.1164/rccm.200703-456SO

6. Tan WC, Bourbeau J, FitzGerald JM, et al. Can age and sex explain the variation in COPD rates across large urban cities? A population study in Canada. Int J Tuberc Lung Dis. 2011;15(12):1691-1698. doi:10.5588/ijtld.11.0211
7. Bourbeau J, Tan WC, Benedetti A, et al. Canadian cohort obstructive lung disease (CanCOLD): fulfilling the need for longitudinal observational studies in COPD. COPD. 2014;11(2):125-132. doi:10.3109/ 15412555.2012 .665520

8. Janz $\mathrm{T}$ Current smoking trends. Ottawa, ON, Canada: Statistics Canada Catalogue; 2015. Available from: https://www150.statcan. gc.ca/n1/pub/82-624-x/2012001/article/11676-eng.htm. Accessed November 13, 2020.

9. Buist AS, McBurnie MA, Vollmer WM, et al. International variation in the prevalence of COPD (the BOLD Study): a population-based prevalence study. Lancet Lond Engl. 2007;370(9589):741-750. doi:10.1016/S0140-6736(07)61377-4

10. Buist AS, Vollmer WM, Sullivan SD, et al. The burden of obstructive lung disease initiative (BOLD): rationale and design. COPD. 2005;2 (2):277-283. doi:10.1081/COPD-57610

11. Menezes AMB, Perez-Padilla R, Jardim JRB, et al. Chronic obstructive pulmonary disease in five Latin American cities (the PLATINO study): a prevalence study. Lancet Lond Engl. 2005;366 (9500):1875-1881. doi:10.1016/S0140-6736(05)67632-5

12. Miller MR. Standardisation of spirometry. Eur Respir J. 2005;26 (2):319-338. doi:10.1183/09031936.05.00034805

13. Tan WC, Bourbeau J, O'Donnell D, et al. Quality assurance of spirometry in a population-based study -predictors of good outcome in spirometry testing. COPD. 2014;11(2):143-151. doi:10.3109/ 15412555.2013 .822857

14. Singh D, Agusti A, Anzueto A, et al. Global strategy for the diagnosis, management, and prevention of chronic obstructive lung disease: the GOLD science committee report 2019. Eur Respir J. 2019;53(5). doi:10.1183/13993003.00164-2019

15. Celli BR, Halbert RJ, Isonaka S, Schau B. Population impact of different definitions of airway obstruction. Eur Respir J. 2003;22 (2):268-273. doi:10.1183/09031936.03.00075102

16. Swanney MP, Ruppel G, Enright PL, et al. Using the lower limit of normal for the FEV1/FVC ratio reduces the misclassification of airway obstruction. Thorax. 2008;63(12):1046-1051. doi:10.1136/ thx.2008.098483

17. Hankinson JL, Odencrantz JR, Fedan KB. Spirometric reference values from a sample of the general U.S. population. Am J Respir Crit Care Med. 1999;159(1):179-187. doi:10.1164/ajrccm.1 59.1.9712108

18. Vollmer WM, Gislason T, Burney P, et al. Comparison of spirometry criteria for the diagnosis of COPD: results from the BOLD study. Eur Respir J. 2009;34(3):588-597. doi:10.1183/ 09031936.00164608

19. Deeks JJ, Higgins JP; Altman DG on behalf of the Cochrane Statistical Methods Group. Chapter 9: analysing data and undertaking meta-analyses. In: Higgins JP, Green S, editors. Cochrane Handbook for Systematic Reviews of Interventions: Cochrane Book Series. Chichester, UK: John Wiley \& Sons; 2008.

20. Health characteristics, annual estimates table 13-10-0096-01. Ottawa, ON, Canada: Statistics Canada; 2020. Available from: https:// www150.statcan.gc.ca/t1/tbl1/en/tv.action?pid=1310009601. Accessed February 15, 2020.

21. Gershon AS, Wang C, Guan J, Vasilevska-Ristovska J, Cicutto L, To T. Identifying individuals with physcian diagnosed COPD in health administrative databases. COPD. 2009;6(5):388-394. doi:10.1080/15412550903140865

22. Ospina MB, Voaklander D, Senthilselvan A, et al. Incidence and prevalence of chronic obstructive pulmonary disease among aboriginal peoples in Alberta, Canada. PLoS One. 2015;10(4):e0123204. doi:10.1371/journal.pone.0123204

23. Evans J, Chen Y, Camp PG, Bowie DM, McRae L. Estimating the prevalence of COPD in Canada: reported diagnosis versus measured airflow obstruction. Health Rep. 2014;25(3):3-11. 
24. Labonté LE, Tan WC, Li PZ, et al. Undiagnosed chronic obstructive pulmonary disease contributes to the burden of health care use. Data from the CanCOLD study. Am J Respir Crit Care Med. 2016;194 (3):285-298. doi:10.1164/rccm.201509-17950C

25. Halbert RJ, Natoli JL, Gano A, Badamgarav E, Buist AS, Mannino DM. Global burden of COPD: systematic review and meta-analysis. Eur Respir J. 2006;28(3):523-532. doi:10.1183/ 09031936.06 .00124605

26. Aaron SD, Gershon AS, Gao Y, Yang J, Whitmore GA. Influence of country-level differences on COPD prevalence. Int J Chron Obstruct Pulmon Dis. 2016;11:2305-2313. doi:10.2147/COPD.S113868

27. Vogelmeier CF, Criner GJ, Martinez FJ, et al. Global strategy for the diagnosis, management, and prevention of chronic obstructive lung disease 2017 report. GOLD executive summary. Am J Respir Crit Care Med. 2017;195(5):557-582. doi:10.1164/rccm.201701-0218PP

28. Postma DS, Bush A, van den Berge M. Risk factors and early origins of chronic obstructive pulmonary disease. Lancet Lond Engl. 2015;385(9971):899-909. doi:10.1016/S0140-6736(14)60446-3

29. Osman S, Ziegler C, Gibson R, Mahmood R, Moraros J. The association between risk factors and chronic obstructive pulmonary disease in Canada: a cross-sectional study using the 2014 Canadian community health survey. Int J Prev Med. 2017;8:86. doi:10.4103/ijpvm.IJPVM 330_17

30. Lamprecht B, McBurnie MA, Vollmer WM, et al. COPD in never smokers: results from the population-based burden of obstructive lung disease study. Chest. 2011;139(4):752-763. doi:10.1378/ chest.10-1253

31. Paulin LM, Diette GB, Blanc PD, et al. Occupational exposures are associated with worse morbidity in patients with chronic obstructive pulmonary disease. Am J Respir Crit Care Med. 2015;191 (5):557-565. doi:10.1164/rccm.201408-1407OC

32. Eisner MD, Anthonisen N, Coultas D, et al. An official American Thoracic Society public policy statement: novel risk factors and the global burden of chronic obstructive pulmonary disease. Am J Respir Crit Care Med. 2010;182(5):693-718. doi:10.1164/rccm.200811-1757ST

33. Barker DJ, Godfrey KM, Fall C, Osmond C, Winter PD, Shaheen SO. Relation of birth weight and childhood respiratory infection to adult lung function and death from chronic obstructive airways disease. BMJ. 1991;303(6804):671-675. doi:10.1136/bmj.303.6804.671
34. Todisco T, de Benedictis FM, Iannacci L, et al. Mild prematurity and respiratory functions. Eur J Pediatr. 1993;152(1):55-58. doi:10.1007/ BF02072517

35. de Marco R, Accordini S, Marcon A, et al. Risk factors for chronic obstructive pulmonary disease in a European cohort of young adults. Am J Respir Crit Care Med. 2011;183(7):891-897. doi:10.1164/ rccm.201007-1125OC

36. Tan WC, Sin DD, Bourbeau J, et al. Characteristics of COPD in never-smokers and ever-smokers in the general population: results from the CanCOLD study. Thorax. 2015;70(9):822-829. doi:10.1136/thoraxjnl-2015-206938

37. Silva GE, Sherrill DL, Guerra S, Barbee RA. Asthma as a risk factor for COPD in a longitudinal study. Chest. 2004;126(1):59-65. doi:10.1378/chest.126.1.59

38. Aryal S, Diaz-Guzman E, Mannino DM. Influence of sex on chronic obstructive pulmonary disease risk and treatment outcomes. Int J Chron Obstruct Pulmon Dis. 2014;9:1145-1154. doi:10.2147/ COPD.S54476

39. Sørheim I-C, Johannessen A, Gulsvik A, Bakke PS, Silverman EK, DeMeo DL. Gender differences in COPD: are women more susceptible to smoking effects than men? Thorax. 2010;65(6):480-485. doi:10.1136/thx.2009.122002

40. Vanfleteren LEGW, Franssen FME, Wesseling G, Wouters EFM. The prevalence of chronic obstructive pulmonary disease in Maastricht, the Netherlands. Respir Med. 2012;106(6):871-874. doi:10.1016/j. rmed.2012.01.008

41. Bird Y, Moraros J, Mahmood R, Esmaeelzadeh S, Kyaw Soe NM. Prevalence and associated factors of COPD among Aboriginal peoples in Canada: a cross-sectional study. Int J Chron Obstruct Pulmon Dis. 2017;12:1915-1922. doi:10.2147/COPD.S138304

42. Ntritsos G, Franek J, Belbasis L, et al. Gender-specific estimates of COPD prevalence: a systematic review and meta-analysis. Int J Chron Obstruct Pulmon Dis. 2018;13:1507-1514. doi:10.2147/COPD.S146390

43. Khan M, Kobayashi K, Lee S, Vang Z. (In)Visible minorities in canadian health data and research. Popul Change Lifecourse Strateg Knowl Clust Discuss Pap Ser Un Réseau Strat Connaiss Chang Popul Parcours Vie Doc Trav. 2015;3(1):5.

\section{Publish your work in this journal}

The International Journal of COPD is an international, peer-reviewed journal of therapeutics and pharmacology focusing on concise rapid reporting of clinical studies and reviews in COPD. Special focus is given to the pathophysiological processes underlying the disease, intervention programs, patient focused education, and self management protocols. This journal is indexed on PubMed Central, MedLine and CAS. The manuscript management system is completely online and includes a very quick and fair peer-review system, which is all easy to use. Visit http://www.dovepress.com/testimonials.php to read real quotes from published authors.

Submit your manuscript here: https://www.dovepress.com/international-journal-of-chronic-obstructive-pulmonary-disease-journal 